\title{
Sequence and structural relationships of leucocins A-, B- and C-TA33a from Leuconostoc mesenteroides TA33a
}

\author{
Maria A. Papathanasopoulos, ${ }^{1,2}$ Gary A. Dykes, ${ }^{1}$ \\ Anne-Marie Revol-Junelles, ${ }^{3}$ Antoine Delfour, ${ }^{4}$ \\ Alexander von Holy ${ }^{2}$ and John W. Hastings ${ }^{1}$
}

Author for correspondence: John W. Hastings. Tel: +27 331260 5434. Fax: +27 3312605435. e-mail: hastings@gene.unp.ac.za

1 Department of Genetics, University of Natal, Pietermaritzburg, Private Bag X01, Scottsville, 3209, South Africa

2 Department of Microbiology, University of the Witwatersrand, Private Bag 3, WITS, 2050, South Africa

3 Laboratoire de Fermentations et de Bioconversions Industrielles, ENSAIA, Vandoeuvre les Nancy, France

4 IBMIG, Laboratoire de Biochimie des Proteines, Université de Poitiers, France

\begin{abstract}
Amino acid sequences of two of the three bacteriocins from Leuconostoc mesenteroides TA33a were determined and their sequence-structure relationships investigated. Leucocin B-TA33a consists of 31 amino acid residues, with a molecular mass of $3466 \mathrm{Da}$. Leucocin B-TA33a does not belong to the pediocin family of bacteriocins, but shares $62 \%$ homology with mesenterocin 52B. A partial sequence of 36 amino acids of leucocin C-TA33a (4598 Da) was determined. Leucocin C-TA33a belongs to the class II bacteriocins having the consensus YGNGV motif. The third bacteriocin, leucocin A-TA33a, is identical to leucocin A-UAL 187. Circular dichroism spectra of the leucocins in aqueous solution and micellar SDS indicated that they undergo a structural transition when in a membrane-mimicking environment. Theoretical predictions from circular dichroism data suggest that leucocins A-, B- and CTA33a adopt a $\beta$-structure (48\%) in membrane-mimicking environments. Sequence alignments and secondary structure predictions for the $\mathrm{N}$-terminus of leucocins A- and C-TA33a predicted that Cys-9 and Cys-14 are connected by a disulfide bridge and form two $\beta$-strands.
\end{abstract}

Keywords: bacteriocins, leucocins, circular dichroism, secondary structure, Leuconostoc mesenteroides

\section{INTRODUCTION}

Bacteriocins of lactic acid bacteria are ribosomally synthesized antimicrobial peptides, which may inhibit their target cells by permeabilizing the cell membrane (Chen et al., 1997; Fleury et al., 1996; Klaenhammer, 1993 ; Nissen-Meyer \& Nes, 1997). Their positive charge presumably facilitates interactions with the negatively charged bacterial phospholipid-containing membranes and/or acidic bacterial cell walls, whereas their amphiphilic character enables membrane permeabilization (Nissen-Meyer \& Nes, 1997). Class I bacteriocins, for example nisin, undergo extensive post-translational modifications and contain unusual amino acids such as lanthionine and 3-methyllanthionine (Klaenhammer,

Abbreviation: $\mathrm{CD}$, circular dichroism.

The SWISS-PROT accession numbers for the amino acid sequences of leucocins B- and C-TA33a reported in this paper are P81052 and P81053, respectively.
1993). By contrast, the class II bacteriocins are minimally modified and have high amino acid sequence homologies. Class II bacteriocins include: carnobacteriocin BM1 and B2 (Quadri et al., 1994), curvacin A (Tichaczek et al., 1993), enterocin A (Aymerich et al., 1996), lactococcin G (Nissen-Meyer et al., 1992), leucocin AUAL 187 (Hastings et al., 1991), leucocin B-TA11a (Felix et al., 1994), mesentericin Y105 (Héchard et al., 1992), mesentericin Y105 ${ }^{37}$ (Fleury et al., 1996), mesenterocin FR52A (Revol-Junelles et al., 1996), pediocin PA-1 (Henderson et al., 1992), piscicolin 126 (Jack et al., 1996), piscicolin 61 (Holck et al., 1994), sakacin A (Holck et al., 1992) and sakacin P (Tichaczek et al., 1994).

Since the biologically relevant conformation of bacteriocins is the one adopted in a lipophilic environment, solvent-dependent circular dichroism (CD) and NMR can be used to gain insight into their conformational transitions in aqueous and lipophilic environments. 
Little is known about the secondary and tertiary structures of class II bacteriocins, except for leucocin AUAL 187, mesentericin Y $105^{37}$ and lactococcin G (Fleury et al., 1996; Henkel et al., 1992; Nissen-Meyer \& Nes, 1997; Sailer et al., 1993). Data from NMR studies indicate that leucocin A-UAL 187 exists as a random coil in water and changes in membrane-mimicking solvents to form an amphiphilic helical region in the C-terminal region after the loop maintained by the disulfide bond (Henkel et al., 1992; Sailer et al., 1993). CD spectra of mesentericin $\mathrm{Y}^{105^{37}}$ in a low polarity medium indicated $30-40 \% \alpha$-helical conformation and secondary structure predictions suggested the peptide can be configured as an amphipathic helix at the C-terminus (Fleury et al., 1996). The two peptides that constitute lactococcin $G$ contain regions that become amphiphilic if they assume an $\alpha$-helical structure, and CD studies have shown that these regions adopt an $\alpha$-helical structure when the two peptides interact with each other in the presence of membrane-mimicking micelles (Nissen-Meyer \& Nes, 1997). CD and NMR studies on the lantibiotics nisin (Van den Hooven et al., 1993) and Pep5 (Freund et al., 1991), showed that these peptides exhibit a flexible, unordered structure in aqueous solution and several constrained regions in membrane-mimicking environments. The major conformational changes for nisin occur at the N-terminus.

Recently, Leuconostoc mesenteroides TA33a was found to produce three different bacteriocins with different antimicrobial activity spectra (Papathanasopoulos $e t a l$., 1997). This paper reports on the primary structures of leucocins A-, B- and C-TA33a produced by L. mesenteroides TA33a and a comparison of the conformations adopted by these peptides in membrane-mimicking environments.

\section{METHODS}

Bacterial strains and media. The bacteriocin producer $L$. mesenteroides TA33a and the indicator strain Weissella paramesenteroides DSM 20288, were grown to late-exponential phase at $25^{\circ} \mathrm{C}$ for $18-20 \mathrm{~h}$ in MRS broth (Biolab). The indicator strains Carnobacterium mobile DSM 4848 and Enterococcus durans CIP 55125 were grown to late-exponential phase at $30^{\circ} \mathrm{C}$ for $18-20 \mathrm{~h}$ in Tryptone Soy broth (Biolab).

Bacteriocin activity assay. Bacteriocin activity was assayed using the agar well diffusion method, as described previously (Papathanasopoulos et al., 1997; Revol-Junelles et al., 1996).

Bacteriocin purification. Leucocins A-, B- and C-TA33a were purified by adsorption/desorption of bacteriocins from the producer cells of $L$. mesenteroides TA33a, followed by reversed-phase HPLC, as described previously (Papathanasopoulos et al., 1997; Yang et al., 1992). Aliquots of each purified preparation were lyophilized for further analysis. They were examined by Tricine/SDS-PAGE (Schagger \& Von Jagow, 1987) and stained using silver nitrate (Merril, 1990).

Mass spectrometry and amino acid sequence analysis. Peptide samples were analysed for purity by mass spectrometry with a VG Biotech (Fisons Instruments) equipped with an electrospray source, before their amino acid sequences were determined by Edman degradation ( $250 \mathrm{pmol}$ of each sample) using a 476A Applied Biosystems-Perkin Elmer gasphase sequencer. Phenylthiohydrantoin (PTH) amino acids were identified on line with a $120 \mathrm{~A}$ Applied Biosystems PTH analyser by reversed-phase HPLC. The samples were sequenced using polybrene-coated glass fibre as support. A FASTA search of the SWISS-PROT database was conducted to identify proteins with sequences similar to those of leucocins A-, B- and C-TA33a (Altschul et al., 1990). Multiple alignments were performed using CLUSTAL $\mathrm{W}$ version 1.6.

CD measurements. Leucocins A-, B- and C-TA33a were each resuspended in $10 \mathrm{mM}$ acetate buffer (pH 3.0), $10 \mathrm{mM}$ Tris/ $\mathrm{HCl}$ buffer $(\mathrm{pH} \mathrm{7.0)}$ and $10 \mathrm{mM}$ micellar SDS. CD spectra were recorded between $180 \mathrm{~nm}$ and $260 \mathrm{~nm}$ for each of the above solutions at room temperature in a quartz cell (path length $1 \mathrm{~mm}$ ) on a J-720 spectropolarimeter (JASCO). Data were digitally collected every $0 \cdot 1 \mathrm{~nm}$ and the resulting spectra smoothed using the JASCO program. In all cases the CD spectra of the solutions were recorded and subtracted from experimental readings to compensate for background scattering. All spectra presented are the means of five scans. Results are expressed in terms of mean residue ellipticity.

Analysis for secondary structure. The CD spectra were analysed for secondary structure between $180 \mathrm{~nm}$ and $260 \mathrm{~nm}$ using the variable selection subroutine (Johnson, 1990; Manavalan \& Johnson, 1987) of the DicoProt program (G. Dileage, Institute de Biologie et Chimie des Proteines, Lyon, France). In addition, secondary structure was estimated from spectra between $200 \mathrm{~nm}$ and $240 \mathrm{~nm}$ using the K2D CD secondary structure server, which uses an unsupervised neural network to predict secondary structure (Andrade et al., 1993; Merelo et al., 1994).

\section{RESULTS AND DISCUSSION}

\section{Mass spectrometry and amino acid sequence analysis}

Analysis of the purified peptides by Tricine/SDS-PAGE followed by silver nitrate staining revealed single bands for each peptide in the $2.5 \mathrm{kDa}$ size range (Fig. 1), indicating that the bacteriocins had been purified to homogeneity. Mass spectrometry predicted masses of 3930,3467 and $4598 \mathrm{Da}$ for leucocins A-, B- and CTA33a, respectively.

The primary structure of purified leucocin B-TA33a was successfully determined up to the thirty-first residue as KGKGFWSWASKATSWLTGPQQPGSPLLKKHR

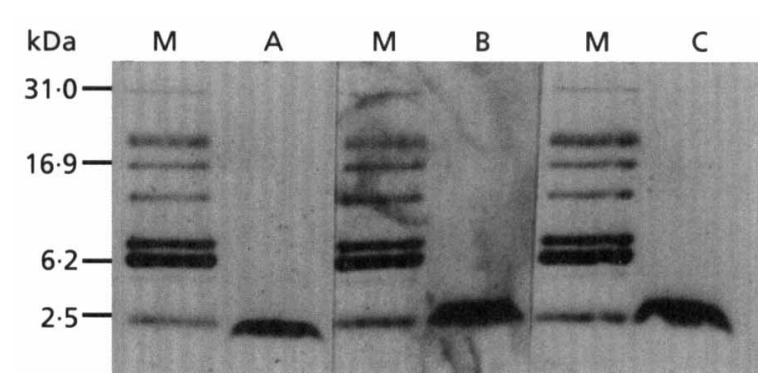

Fig. 1. Tricine/SDS-PAGE analysis of purified leucocins A-, B- and C-TA33a. Lanes: $M$, low-range molecular mass markers (Promega); A, purified leucocin A-TA33a; B, purified leucocin BTA33a; C, purified leucocin C-TA33a. 


\begin{tabular}{|c|c|c|}
\hline Bacteriocin & Sequence & acids \\
\hline Leucocin C-TA33a & - - - - KNYGNG-VECTKKGCSVDWGYAAT - - -NIALNSVMNGLTG - - - - - & 36 \\
\hline Sakacin P & - - - - KYYGNG-VECGKHSCTVDWGTAIG - - -NIGNNAAANWATGGNAGWNK - & 43 \\
\hline Bavaricin A & - - - - KYYGNG-VHXGRHSXTVDWGTAIG - - -NIGNAAAANXATGXNAGG - - & 41 \\
\hline Piscicolin 126 & - - - - KYYGNG-VSCNRNGCTVDWSKAIG-- - I IGNNAAANLTTGGAAGWNKG & 44 \\
\hline Pediocin PA-1 & - - - - KYYGNG-VTCGKHSCSVDWGKATT - - - CIINNGAMAWATGGHQGNHKC & 44 \\
\hline Enterocin A & TTHSGKYYGIG-VYCTKNKCTVDWAKATT - - - - CIAGSMIGGFLGGAIPGKC - - & 47 \\
\hline Leucocin A-UAL 187 & - - - - KYYGNG - VHCTKSGCSVINWGEAFS - - -AGVHRLANGGNGFW - - - - - & 37 \\
\hline Leucocin B-TA11a & - - - -RYYGNG-VHCTKSGCSVNWGEAFS - - - -AGVHRLANGGNGFW- - - - - - & 37 \\
\hline Mesentericin Y105 & - - - - KYYGNGVVHCTKSGCSVNWGEAAS - - - AGIHRLANGGNGFW - - - - - & 38 \\
\hline Sakacin A & - - - ARSYGNG-VYCNNKKCWVNRGEATQ- - - SIIGGMISGWASGLAGM - - - & 41 \\
\hline Carnobacteriocin BM1 & - - - AISYGWG-VYCNKEKCWVNKAENKQ- - -AITGIVIGGWASSLAGMGH- - & 43 \\
\hline Carnobacteriocin B2 & - - - - VNYGNG-VSCSKTKCSVNWGQAFQERYTAGINSFVSGVASGAGS IGRRP & 48 \\
\hline
\end{tabular}

Fig. 2. Multiple alignment of the amino acid sequence of leucocin C-TA33a and other class II bacteriocins. Dashes represent gaps introduced to optimize the alignment and consensus amino acids are indicated in bold type. References for sequences are as follows: sakacin P, Tichaczek et al. (1994); bavaricin A, Larsen et al. (1993); piscicolin 126, Jack et al. (1996); pediocin PA-1, Henderson et al. (1992); enterocin A, Aymerich et al. (1996); leucocin A-UAL 187 (identical to leucocin A-TA33a), Hastings et al. (1991); leucocin B-TA11a, Felix et al. (1994); mesentericin Y105 (identical to mesenterocin FR52A), Héchard et al. (1992); sakacin A, Holck et al. (1992); carnobacteriocin BM1 and B2, Quadri et al. (1994).

by automated Edman degradation of the peptide $(250 \mathrm{pmol})$. The calculated molecular mass of leucocin B-TA33a was $3466 \mathrm{Da}$. The discrepancy of $1 \mathrm{Da}$ between the calculated and measured molecular masses resulted from the presence of hydrogen. The partial amino acid sequence of leucocin C-TA33a was determined up to the thirty-sixth residue as KNYGNGVHCTKKGCSVDWGYAATNIANNSVMNGLTG (calculated molecular mass of $3744 \mathrm{Da}$ ). The amino acid sequence of leucocin A-TA33a has previously been determined by translation of the nucleotide sequence (GenBank accesion number AFO36713) and found to be identical to that of leucocin A-UAL 187 (KYYGNGVHCTKSGCSVNWGEAFSAGVHRLANGGNGFW).

All three leucocins are strongly hydrophobic. Leucocins A- and C-TA33a have similar hydropathy profiles at the $\mathrm{N}$-terminus of the peptide, but differ at the C-terminus. Leucocin B-TA33a appears to be the most hydrophobic. The hydrophilic $\mathrm{N}$-terminal region of leucocin C-TA33a contains two cysteines at positions 9 and 14 (as leucocin A-TA33a) and these are likely to be joined by a disulfide bridge. The $\mathrm{N}$-terminus also contains the consensus motif YGNGVXCXK/NXXC, where $X$ represents polar uncharged or charged residues. Leucocin C-TA33a shares strong homology to class II bacteriocins other than those produced by Leuconostoc strains. The partial sequence of leucocin C-TA33a shares 80 and $78 \%$ homology with leucocin A-UAL 187 (as well as leucocin A-TA33a and leucocin B-TA11a) and mesentericin Y105, respectively; 70 and $56 \%$ with carnobacteriocin B2 and BM1, respectively; $61 \%$ homology with sakacin $\mathrm{P}$ and pediocin PA-1; $55 \%$ with piscicolin 126, bavaricin $A$ and enterocin A; and $36 \%$ with sakacin A (curvacin A). Leucocin C-TA33a appears to be similar to other Leuconostoc bacteriocins at the $\mathrm{N}$-terminus, but more similar to other known class II bacteriocins at the Cterminus. Multiple sequence alignments of class II bacteriocins sharing homology with leucocin C-TA33a are shown in Fig. 2.

The sequence of leucocin B-TA33a is $62 \%$ homologous to that of the 32 residue peptide mesentericin 52B (Revol-Junelles et al., 1996) and 63\% homologous to the partially sequenced dextranicin 24 (Revol-Junelles \& Lefebvre, 1996).

\section{CD spectra of leucocins}

The influence of $\mathrm{pH}$ and membrane-mimicking environments on the conformation of leucocins A-, B- and C-TA33a was examined by $\mathrm{CD}$ spectrometry. Conformations at $\mathrm{pH} 3$ and 7 were identical (results not shown), indicating that the structure is probably unchanged. The spectra of all three leucocins changed in micellar SDS, suggesting conformational transitions 

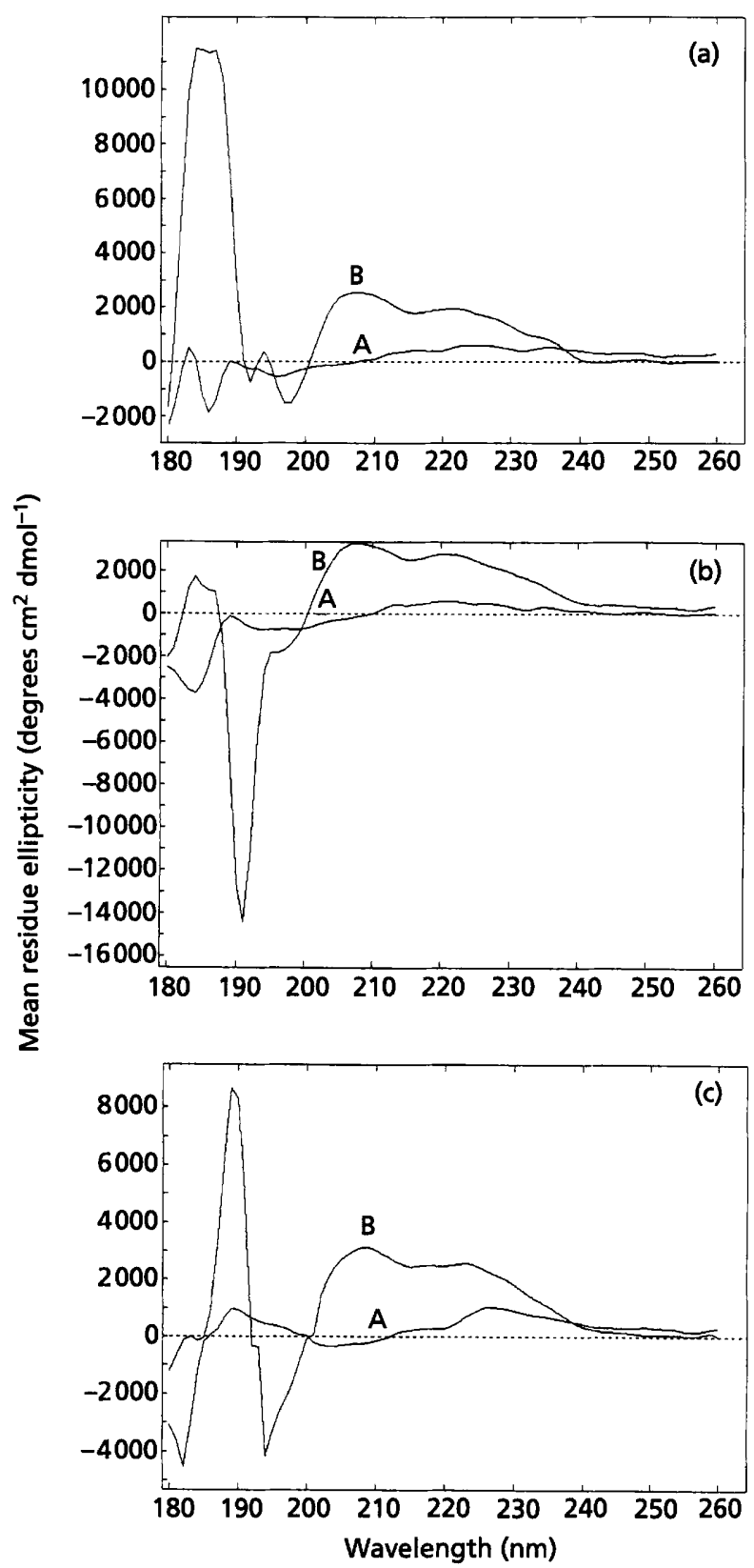

Fig. 3. Comparison of CD spectra of leucocin A-TA33a (a); leucocin B-TA33a (b); and leucocin C-TA33a (c) in aqueous solution (A) and micellar SDS (B).

(Fig. 3a-c). The conformation is strongly dependent on the polarity of the environment. In water, leucocins seem to have a less ordered structure, whereas they appear to be more rigid in membrane-mimicking environments. All three leucocins appear to have CD spectra more typical of $\beta$-structures than $\alpha$-helices (Woody, 1995). The peptides have negative bands at $180 \mathrm{~nm}$ $(\beta$-sheet) and positive bands between 200 and $210 \mathrm{~nm}$ $(\beta$-turn).

The percentages of each leucocin molecule that adopt a particular secondary structure in aqueous and micellar
Table 1. Estimation of the percentage of peptide in a given secondary structure in aqueous solution and $10 \mathrm{mM}$ micellar SDS, determined using the K2D CD secondary structure server

$\mathrm{H}, \alpha$-helix; B, total $\beta$-content (strand and turn); R, random coil.

\begin{tabular}{|llrrr|}
\hline Peptide & Solvent & $\% \mathbf{H}$ & $\% \mathrm{~B}$ & $\% \mathrm{R}$ \\
\hline Leucocin A-TA33a & Aqueous & 4 & 48 & 48 \\
& SDS & 4 & 48 & 48 \\
Leucocin B-TA33a & Aqueous & 37 & 26 & 38 \\
& SDS & 4 & 48 & 48 \\
Leucocin C-TA33a & Aqueous & 4 & 48 & 48 \\
& SDS & 4 & 48 & 48 \\
\hline
\end{tabular}

SDS solutions were estimated using the K2D and variable selection methods (Table 1 ). According to the K2D method, all three leucocins adopt a high degree of $\beta$-sheet (strand and turn) conformation in micellar SDS. Leucocins A- and C-TA33a appear to maintain the same percentages of each conformation in both aqueous and lipophilic environments, whilst leucocin B-TA33a shifts from a more evenly distributed conformation in aqueous solution to a predominantly $\beta$-sheet conformation in micellar SDS. The variable selection method could not further resolve the total J-content into $\beta$-strands (parallel and antiparallel) and J-turns.

Information from $C D$ was used in conjunction with secondary structure prediction algorithms based on protein sequences. A consensus secondary structure prediction for all leucocins, based on six methods (Deleage \& Roux, 1987; Geourjon \& Deleage, 1995; Gibrat et al., 1987; Kneller et al., 1990; Levin et al., 1986; Rost \& Sander, 1994) is presented in Fig. 4. All methods used to predict secondary structure for leucocin A- and C-TA33a identified three short $\beta$-strands at the $\mathrm{N}$-terminus at approximately the same positions. The sequence fragments containing Cys-9 and Cys-14 form two $\beta$-strands, connected by a short loop or turn. Biochemical studies suggest that the cysteine side-chains found in class II bacteriocins are linked by a disulfide bridge (Chen et al., 1997; Fleury et al., 1996). The sixmembered disulfide loops linking Cys-9 and Cys-14 in leucocins A- and C-TA33a should impose a compact local structure. This is in agreement with the results of NMR data for leucocin A-UAL 187, which show that leucocin A-UAL 187 adopts a rigid conformation for residues near the disulfide bridge in membrane-mimicking environments (Henkel et al., 1992). Furthermore, in membrane-mimicking solvents an amphiphilic helical region is present in the C-terminal section of leucocin AUAL 187 after the loop maintained by the disulfide bond (Sailer et al., 1993). Similar regions were identified in mesentericin $\mathrm{Y}^{105^{37}}$ using theoretical predictions and $\mathrm{CD}$ measurements, with the amphipathic helix spanning residues 17-31 (Fleury et al., 1996). Secondary structure predictions for leucocin A-TA33a exhibit an $\alpha$-helix 


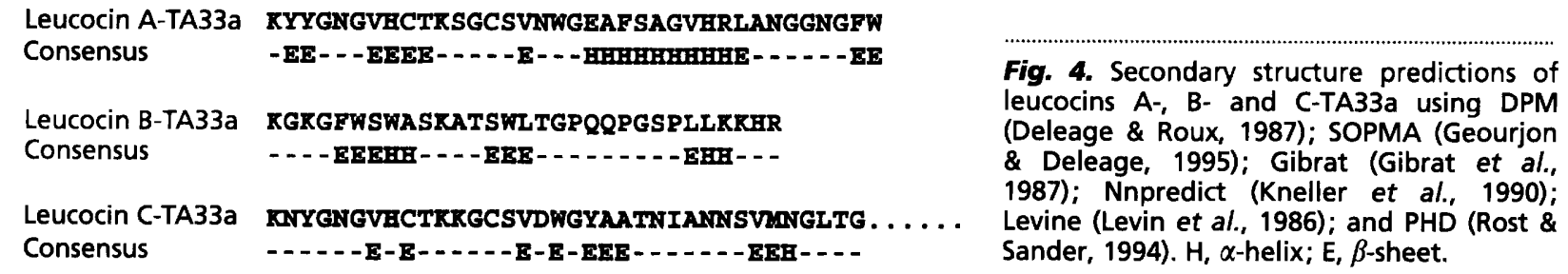

stretch towards the C-terminus of the peptide, which contains both hydrophobic and hydrophilic residues and spans residues $20-28$. The propensity of small-sized cationic peptides to form helical amphipathic structures in apolar media has been proposed as a prerequisite for their membrane-disrupting activity (Nissen-Meyer $\&$ Nes, 1997). Secondary structure predictions for leucocins B- and C-TA33a identified $\beta$-strands throughout the length of the peptide.

Similar results were obtained from secondary structure predictions for the N-terminus of pediocin PA-1 and other class II bacteriocins (Chen et al., 1997). The Nterminus of pediocin PA-1 contained two $\beta$-sheets maintained in a hairpin conformation stabilized by a disulfide bridge. Except for the second disulfide bond (Cys-24 and Cys-44), no structure could be predicted for the C-terminus of pediocin PA- 1 because of a high degree of conformational freedom. The structural model also revealed patches of positively charged residues, which could allow the bacteriocin to interact directly with Listeria lipid vesicles which contain anionic phospholipid head groups (Chen et al., 1997). The tip of the $\beta$-hairpin created by the disulfide bond in leucocins Aand C-TA33a contains one (Lys-11) and two (Lys-11 and Lys-12) positive residues, respectively.

The occurrence of structural changes in environments that mimic the site of action of the bacteriocins suggests that they have some inherent flexibility, and insertion of the bacteriocin into bacterial membranes may be accompanied by an essential alteration in its secondary structure.

\section{ACKNOWLEDGEMENTS}

This work was partly supported by the Foundation for Research and Development of South Africa. Grateful thanks are extended to F. Krier for his technical assistance.

\section{REFERENCES}

Altschul, S. F., Gish, W., Miller, W., Myers, E. W. \& Lipman, D. J. (1990). Basic local alignment search tool. J Mol Biol 215, 403-410.

Andrade, M. A., Chacon, P., Merelo, J. J. \& Moran, F. (1993). Evaluation of secondary structure of proteins from UV circular dichroism spectra using an unsupervised learning neural network. Protein Eng 6, 383-390.

Aymerich, T., Holo, H., Havarstein, L. S., Hugas, M., Garriga, M. \& Nes, I. F. (1996). Biochemical and genetic characterization of enterocin A from Enterococcus faecium, a new antilisterial bacteriocin in the pediocin family of bacteriocins. Appl Environ Microbiol 62, 1676-1682.

Chen, Y., Shapira, R., Eisenstein, M. \& Montville, T. J. (1997). Functional characterization of pediocin PA-1 binding to liposomes in the absence of a protein receptor and its relationship to a predicted tertiary structure. Appl Environ Microbiol 63, 524-531.

Deleage, G. \& Roux, B. (1987). An algorithm for protein secondary structure prediction based on class prediction. Protein Eng 1, 289-294.

Felix, J. V., Papathanasopoulos, M. A., Smith, A. A., von Holy, A. \& Hastings, J. W. (1994). Characterization of leucocin B-TA11a: a bacteriocin from Leuconostoc carnosum TA11a isolated from meat. Curr Microbiol 29, 207-212.

Fleury, Y., Dayem, M. A., Montagne, J. J., Chaboisseau, E., Le Caer, J. P., Nicolas, P. \& Delfour, A. (1996). Covalent structure, synthesis, and structure-function studies of mesentericin $\mathrm{Y}_{105^{37}}$, a defensive peptide from Gram-positive bacteria Leuconostoc mesenteroides. J Biol Chem 271, 14421-14429.

Freund, S., Jung, G., Gibbons, W. A. \& Sahl, H.-G. (1991). NMR and circular dichroism studies on Pep5. In Nisin and Novel Lantibiotics, pp. 103-112. Edited by G. Jung \& H.-G. Sahl. Leiden : ESCOM.

Geourjon, C. \& Deleage, G. (1995). sopMA: significant improvements in protein secondary structure prediction from multiple alignments. Comput Appl Biosci 11, 681-684.

Gibrat, J. F., Garnier, J. \& Robson, B. (1987). Further developments of protein secondary structure prediction using information theory: new parameters and consideration of residue pairs. $J \mathrm{Mol}$ Biol 198, 425-443.

Hastings, J. W., Sailer, M., Johnson, K., Roy, K. L., Vederas, J. C. \& Stiles, M. E. (1991). Characterization of leucocin A-UAL 187 and cloning of the bacteriocin gene from Leuconostoc gelidum. J Bacteriol 173, 7491-7500.

Héchard, Y., Dérijard, B., Lettelier, F. \& Cenatiempo, Y. (1992). Characterization and purification of mesentericin Y105, an antiListeria bacteriocin from Leuconostoc mesenteroides. J Gen Microbiol 138, 2725-2731.

Henderson, J. T., Chopko, A. L. \& Wassenaar, P. D. (1992). Purification and primary structure of pediocin PA-1 produced by Pediococcus acidilactici PAC1.0. Arch Biochem Biophys 295, 5-12.

Henkel, T., Sailer, M., Helms, G. L., Stiles, M. E. \& Vederas, J. C. (1992). NMR assignment of leucocin A, a bacteriocin from Leuconostoc gelidum, supported by a stable isotope labelling technique for peptides and proteins. J Am Chem Soc 114, 1898-1900. 
Holck, A., Axelsson, L., Birkeland, S.-E., Aukrust, T. \& Blom, H. (1992). Purification and amino acid sequence of sakacin $A$, a bacteriocin from Lactobacillus sake Lb 706. J Gen Microbiol 138, 2715-2720.

Holck, A. L., Axelsson, L. \& Schillinger, U. (1994). Purification and cloning of piscicolin 61, a bacteriocin from Carnobacterium piscicola LV61. Curr Microbiol 29, 63-68.

Jack, R. W., Wan, J., Gordon, J., Harmark, K., Davidson, B. E. \& Hillier, A. J. (1996). Characterization of the chemical and antimicrobial properties of piscicolin 126 , a bacteriocin produced by Carnobacterium piscicola JG126. Appl Environ Microbiol 62, 2897-2903.

Johnson, W. C. (1990). Protein secondary structure and circular dichroism - a practical guide. Proteins 7, 205-214.

Klaenhammer, T. R. (1993). Genetics of bacteriocins produced by lactic acid bacteria. FEMS Microbiol Rev 12, 39-86.

Kneller, D. G., Cohen, F. E. \& Langridge, R. (1990). Improvement in protein secondary structure prediction by an enhanced neural network. J Mol Biol 214, 171-182.

Larsen, A. G., Vogensen, F. K. \& Josephsen, J. (1993). Antimicrobial activity of lactic acid bacteria isolated from sour doughs: purification and characterization of bavaricin A, a bacteriocin produced by Lactobacillus bavaricus M1401. I Appl Bacteriol 75, 113-122.

Levin, J. M., Robson, B. \& Garnier, J. (1986). An algorithm for secondary structure determination in proteins based on sequence similarity. FEBS Lett 205, 303-308.

Manavalan, P. \& Johnson, W. C. (1987). Variable selection method improves the prediction of protein secondary structure from circular dichroism spectra. Anal Biochem 167, 76-85.

Merelo, J. J., Andrade, M. A., Prieto, A. \& Moran, F. (1994). Proteinotopic feature maps. Neurocomputing 6, 443-454.

Merril, C. R. (1990). Gel-staining techniques. Methods Enzymol $182,477-488$.

Nissen-Meyer, J. \& Nes, I. F. (1997). Ribosomally synthesized antimicrobial peptides: their function, structure, biogenesis, and mechanism of action. Arch Microbiol 167, 67-77.

Nissen-Meyer, J., Holo, H., Havarstein, L. S., Sletten, K. \& Nes, I. F. (1992). A novel lactococcal bacteriocin whose activity depends on the complementary action of two peptides. J Bacteriol 174, 5686-5692.

Papathanasopoulos, M. A., Krier, F., Revol-Junelles, A.-M., Lefebvre, G., Le Caer, J. P., von Holy, A. \& Hastings, J. W. (1997). Multiple bacteriocin production by Leuconostoc mesenteroides
TA33a, and other Leuconostoc/Weissella strains. Curr Microbiol 35, 331-335.

Quadri, L. E,, Sailer, M., Roy, K. L., Vederas, J. C. \& Stiles, M. E. (1994). Chemical and genetic characterization of bacteriocins produced by Carnobacterium piscicola LV17B. J Biol Chem 269, 12204-12211.

Revol-Junelles, A.-M. \& Lefebvre, G. (1996). Purification and Nterminal amino acid sequence of dextranicin 24, a bacteriocin of Leuconostoc sp. Curr Microbiol 33, 136-137.

Revol-Junelles, A.-M., Mathis, R., Krier, F., Fleury, Y., Delfour, A. \& Lefebvre, G. (1996). Leuconostoc mesenteroides subsp. mesenteroides FR52 synthesises two distinct bacteriocins. Lett Appl Microbiol 23, 120-124.

Rost, B. \& Sander, C. (1994). Combining evolutionary information and neural networks to predict protein secondary structure. Proteins Struct Funct Genet 19, 55-72.

Sailer, M., Helms, G. L., Henkel, T., Niemczura, W. P., Stiles, M. E. \& Vederas, J. C. (1993). ${ }^{15} \mathrm{~N}$ - and ${ }^{13} \mathrm{C}$-labelled media from Anabaena sp. for universal isotopic labelling of bacteriocins: NMR resonance assignments of leucocin A from Leuconostoc gelidum and nisin A from Lactococcus lactis. Biochemistry 32, 310-318.

Schagger, H. \& Von Jagow, G. (1987). Tricine-sodium dodecylsulphate-polyacrylamide gel electrophoresis for the separation of proteins in the range of 1 to $100 \mathrm{kDa}$. Anal Biochem 166, 368-379.

Tichaczek, P. S., Vogel, R. F. \& Hammes, W. P. (1993). Cloning and sequencing of cur $A$ encoding curvacin $A$, the bacteriocin produced by Lactobacillus curvatus LTH 1174. Arch Microbiol 160, 279-283.

Tichaczek, P. S., Vogel, R. F. \& Hammes, W. P. (1994). Cloning and sequencing of sakP encoding sakacin $\mathrm{P}$, the bacteriocin produced by Lactobacillus sake LTH 673. Microbiology 140, 361-367.

Van den Hooven, H. W., Fogolari, F., Rollema, H. S., Konings, R. N. H., Hilbers, C. W. \& van de Ven, F. J. M. (1993). NMR and circular dichroism studies of the lantibiotic nisin in non-aqueous environments. FEBS Lett 319, 189-194.

Woody, R. W. (1995). Circular dichroism. Methods Enzymol 246, 34-71.

Yang, R., Johnson, M. C. \& Ray, B. (1992). Novel method to extract large amounts of bacteriocins from lactic acid bacteria. Appl Environ Microbiol 58, 3355-3359.

Received 22 September 1997; revised 15 January 1998; accepted 23 January 1998. 